\title{
Does herbal therapy for COVID-19 have implications for forensic practice?
}

\author{
Roger W. Byard ${ }^{1,2}$ (D) lan F. Musgrave ${ }^{1}$
}

Accepted: 6 January 2021 / Published online: 21 January 2021

๑) Springer Science+Business Media, LLC, part of Springer Nature 2021

SARS-CoV-2 (severe acute respiratory syndrome coronavirus 2) is a coronavirus that causes COVID-19 (coronavirus disease 2019). First identified in China in December 2019 it has subsequently been responsible for a pandemic. The rapid spread of the disease with associated morbidity and mortality has had disastrous effects globally. While vaccines and treatment protocols are currently being trialed, the impact on forensic services in certain countries has been profound, requiring the implementation of strict autopsy protocols and guidelines [1].

Another of the dramatic effects of the pandemic has been the proliferation of COVID-related publications, with an estimated 31,360 papers listed on Pubmed to July 2020 [2]. The leading publishing countries were the United States $(5,033)$, China $(3,511)$ and Italy $(2,590)$ with the most popular journals being the British Medical Journal (574), the Journal of Medical Virology (317) and The Lancet (230) [2]. Disturbingly, along with the plethora of publications, referred to as a "paperdemic", there have been a significant number of retracted articles with suggestions of academic mistakes, fraud and dishonesty in the rush to achieve publication status [2,3]. The need for academic integrity and evidence-based research remains paramount [4].

Amongst the many publications now available online are a large number proposing the use of particularly Chinese herbal preparations for both the prevention and treatment of COVID19 [5]. While it is quite likely that certain traditional herbal preparations may alleviate symptoms of infectious diseases such as myalgia and fever, the evidence that herbs can prevent infection with SARS-CoV-2 is less compelling. In addition, the recommendations are often for the use of preparations containing multiple herbs; for example Qingfei Paidu Tang is a combination of four different herbal mixtures containing 21 herbs [5]. It is well recognized that polyherbacy may be

Roger W. Byard

roger.byard@sa.gov.au

1 Adelaide Medical School, The University of Adelaide, Adelaide, SA 5005, Australia

2 Forensic Science SA, Adelaide, SA 5000, Australia associated with a greater risk of adverse reactions [6] and that certain traditional herbal preparations may be incorrectly labelled or adulterated with pharmaceutical agents or toxins [7-9]. Despite this it has been asserted that "no significant adverse effects" were found in a systematic review and metaanalysis of studies of Chinese herbal medicines used to treat COVID-19 in over 700 patients [10]. This is quite surprising, as it appears only logical that a therapeutic agent must have the potential for therapeutic side effects, and yet none were recorded. There is also little independent verification that essential oils can treat COVID-19 by penetrating and disrupting viral membranes [11].

It has been reported that the treatment of COVID-19 patients in Wuhan, China, with traditional Chinese medicines had ">90\% efficacy" and that the combined use of herbal preparations with Western pharmaceuticals is efficacious for certain respiratory symptoms in viral infections [12, 13]. However, it has been pointed out that this has not been supported by "high-quality, rigorously peer-reviewed clinical trials "" "in internationally recognized journals" [14]. There are also risks of adverse herb-drug interactions. For example, a recent in vitro study demonstrated increased hepatotoxicity from acetaminophen (paracetamol) in cell culture in the presence of herbal compounds containing furanocoumarins [15]. As acetaminophen (paracetamol) would be a drug commonly used to treat the symptoms of COVID-19 infection this demonstrates the possibility of adverse interactions. These are all factors that may have an impact on cases that present for medicolegal assessment, although it is yet to be determined how significant this may be.

A review of the literature on the use herbal preparations to treat children with COVID-19 has concluded that there is "no direct evidence on the efficacy of the herbal formulae for the treatment of pediatric COVID-19" [16]. This is an important observation as children should be treated quite circumspectly with herbal preparations as they may be more susceptible to the side effects due to their immature metabolic pathways and physiological processes [17]. 
This editorial is in no way suggesting that traditional herbal preparations may not have a role in treating the symptoms of viral infections, but is instead drawing attention to the fact that herbal preparations do carry sometimes serious side effects and that these can be magnified by polyherbacy and the concomitant use of Western pharmaceuticals. All of this may impact upon medicolegal cases. Despite claims that herbal preparations can prevent COVID-19 infections and carry with them no side effects this has not yet been convincingly demonstrated [14]. There exists, therefore, a very real danger that community members may follow the advice of publications and internet sites that promote unproven theories and herbal use for COVID-19 with a resultant increase, rather than decrease, in therapeutic morbidity and mortality.

\section{References}

1. Santurro A, Scopetti M, D'Errico S, Fineschi V. A technical report from the Italian COVID-19 outbreak. Postmortem sampling and autopsy investigation in cases of suspected or probable COVID19. Forensic Sci Med Pathol. 2020;16:471-6.

2. Boschiero MN, Carvalho TA, Marson FAL. Retraction in the era of COVID-19 and its influence on evidence-based medicine: is science in jeopardy? Pulmonology. 2020;S2531-0437(20):302439. https://doi.org/10.1016/j.pulmoe.2020.10.011.

3. Guzman-Prado Y. Retraction of studies on potential drug therapies for COVID-19: A call for reliability and scientific integrity. Am J Cardiol. 2020;132:173.

4. Dinis-Oliveira RJ. COVID-19 research: pandemic versus "paperdemic", integrity, values and risks of the "speed science". Forensic Sci Res. 2020;5:174-87.

5. Ang L, Lee HW, Choi JY, Zhang J, Lee MS. Herbal medicine and pattern identification for treating COVID-19: a rapid review of guidelines. Int Med Res. 2020;9:100407.
6. Gilbert JD, Musgrave I, Hoban C, Byard RW. Lethal hepatocellular necrosis associated with herbal polypharmacy in a patient with chronic Hepatitis B infection. Forensic Sci Int. 2014;241C:138-40.

7. Byard RW. The potential forensic significance of traditional herbal medicines. J Forensic Sci. 2010;55:89-92.

8. Byard RW, Musgrave I, Maker G, Bunce M. What are the risks to the Australian community from herbal products? Med J Aust. 2017;206:86-90.

9. Coghlan ML, Maker G, Crighton E, Haile J, Murray DC, White NE, et al. Combined toxicological and genetic auditing of traditional Chinese medicines provides a means of detecting adulterants and improving pharmacovigilance. Sci Rep. 2015;5:17475.

10. Fan AY, Gu S, Alemi SF. Chinese herbal medicine for COVID-19: current evidence with systematic review and meta-analysis. J Integ Med. 2020;18:385-94.

11. Asif M, Saleem M, Saadullah M, Yaseen HS, Al ZR. COVID-19 and therapy with essential oils having antiviral, anti-inflammatory, and immunomodulatory properties. Inflammopharmacol. 2020;28:1153-61.

12. Lee DYW, Li QY, Liu J, Efferth T. Traditional Chinese herbal medicine at the forefront battle against COVID-19: Clinical experience and scientific basis. Phytomed. 2021. https://doi. org/10.1016/j.phymed.2020.153337.

13. Li Y, Liu X, Guo L, et al. Traditional Chinese herbal medicine for treating novel coronavirus (COVID-19) pneumonia: protocol for a systemic review and meta-analysis. System Rev. 2020;9:75.

14. Yang Y. Use of herbal drugs to treat COVID-19 should be with caution. Lancet. 2020;395:1689-90.

15. Britza S, Musgrave I, Byard RW. Potential role of herbal traditional Chinese medicines in hepatotoxicity. Leg Med. 2020;47:101740.

16. Ang L, Lee HW, Kim A, Lee JA, Zhang J, Lee MS. Herbal medicines for treatment of children with COVID-19: a review of guidelines. Comp Ther Clin Pract. 2020;39:101174.

17. Farrington R, Musgrave I, Byard RW. Potential implications for the use of herbal preparations in childhood. Acta Paediatr. 2019;108:419-22.

Publisher's Note Springer Nature remains neutral with regard to jurisdictional claims in published maps and institutional affiliations. 